\title{
Barotrauma in COVID-19 Patients
}

\author{
Mohammad Abdallat ${ }^{*}$, Mazhar Khalil', Ghayth Al-Awwa', Ravi Kothuru², Charles La Punzina ${ }^{2}$ \\ 'Department of Surgery, Brookdale University Hospital and Medical Center, USA \\ ${ }^{2}$ Cardiothoracic Surgery, Brookdale University Hospital and Medical Center, USA
}

Article Info

\section{Article Notes}

Received: May 24, 2019

Accepted: June 26, 2020

\section{*Correspondence:}

Mohammad Abdallat, Department of Surgery, Brookdale

University Hospital and Medical Center, USA;

Email: m.abdullat90@gmail.com

C 2020 Abdallat M. This article is distributed under the terms of the Creative Commons Attribution 4.0 International License.

\section{Keywords}

COVID19

Barotrauma

Mechanical ventilation

ARDs

Pneumothorax

case series
Abstract

Background: This case series assessed the clinical outcomes and characteristics of barotrauma in COVID19 patients.

Methods: The electronic medical records of all patients admitted with confirmed COVID 19 infection who eventually developed barotrauma between March $17^{\text {th }}, 2020$ and April $17^{\text {th }}, 2020$ were reviewed, information about patient characteristics, pattern and characteristic of barotrauma were analyzed and reported in a descriptive manner.

Results: 25 patients developed evidence of barotrauma on Chest Xray or Computed tomography (CT) with a mean age of 60.1 at the time of diagnosis, 12 (48\%) developed severe ARDS with PaO2/FiO2 ratio of $<100.14$ (56\%) patients developed pneumothorax, 7 had evidence of subcutaneous emphysema and 6 developed pneumomediastinum. More barotrauma occured in the first day of ventilation than any other day, the median time between mechanical ventilation and development of barotrauma is 3.5 days.

Conclusion: Barotrauma in COVID 19 is associated with an increased mortality (64\%) which may reflect worse acute lung injury in these cases. The median time to develop barotrauma in these patients is similar to the one described in ARDs literature.

\section{Introduction}

Severe acute respiratory syndrome coronavirus 2 (SARSCoV-2) causing coronavirus disease (COVID-19) has been labeled a pandemic by the World Health Organization (WHO), with more than 4.8 million confirmed case and 300 thousand death worldwide to date $^{1}$.

Acute respiratory distress syndrome (ARDS) and hypoxemic respiratory failure associated with COVID-192,3, continues to be a feared complication of this disease. With a case fatality rate ranging $(2-13 \%)^{1,2}$ with the most severe cases requiring intensive care admission and mechanical ventilation and mortality rate reaching $14.6 \%$ for those requiring mechanical ventilation in New York City ${ }^{4}$.

Positive pressure ventilation is a non-physiological and invasive intervention that can be lifesaving in these patients. Similar to any other interventions, it carries its own risk and complication as it can lead to barotrauma and ventilator-induced lung injury, which may be associated with multisystem organ failure (MSOF) in patients with ARDS 5 .

Several studies have shown that using low tidal volume (Vt) and high Positive End Expiratory pressure (PEEP) can decrease Hospital mortality in patients with ARDS $^{6}$. Different guidelines, including the last surviving sepsis campaign guidelines for COVID-19 patients, 
recommends using low Vt $(4-8 \mathrm{ml} / \mathrm{kg})^{7-9}$ as it is believed to decrease the volutrauma in a lung suffering from ARDS.

Other lung-protective strategies include targeting plateau pressures (Pplat) of $<30 \mathrm{~cm} \mathrm{H} 2 \mathrm{O}$ to limit lung injury as Pplat $>32$ were associated with higher short-term mortality ${ }^{10}$. Extrinsic PEEP has been utilized in ARDs to improve oxygenation, reduce Oxygen requirement (FiO2) and prevent Atelectotrauma (repeated opening and closing of alveoli), which reduce Ventilator-induced lung injury (VILI) ${ }^{7}$.

Studies have shown that higher PEEP strategies may improve survival in ARDS, at the possible increase risk of pneumothorax ${ }^{11}$. Increasing the PEEP to sustain adequate oxygenation can be at the expense of increasing Pplat and barotrauma.

Pulmonary barotrauma in mechanical ventilation refers to alveolar rupture due to elevated trans alveolar pressure (the alveolar pressure minus the pressure in the adjacent interstitial space), which leads to air leaks into extra-alveolar tissue that can manifest as pneumothorax, pneumomediastinum, pneumoperitoneum, and subcutaneous emphysema ${ }^{12}$. It is associated with increased morbidity and mortality ${ }^{13,14}$. The incidence of barotrauma varies with the underlying indication for mechanical ventilation, and several previous studies found patients with underlying lung disease (Pneumonia, Chronic interstitial lung disease, ARDs) more prone to barotrauma compared to patients who were intubated for Septic shock or developed ARDs for a non-pulmonary insult ${ }^{15}$. The ARDS net research found that lung-protective ventilation reduces mortality and possibly barotrauma in Acute Lung Injury (ALI) and ARDs ${ }^{7,8}$.

Patients with COVID-19 might have a higher incidence of barotrauma. This incidence is not yet documented, but previous studies have shown that patients with ARDS resulting from pneumonia may develop early barotrauma ${ }^{16}$. These patients usually require high PEEP to maintain oxygenation, which increases their risk for barotrauma. Case reports from China also described spontaneous pneumomediastinum in COVID19 patients $^{17}$.

This paper presents a case series review of barotrauma injuries encountered in the management of COVID-19 cases. It is unique in that it reviews the early experience of a Thoracic Surgery service functioning in the epicenter of the COVID-19 pandemic, the New York City boroughs of Queens and Brooklyn. It encompasses a previously unreported experience with a surprising number of barotrauma complications in COVID-19 patients with experienced severe cases of respiratory failure.

\section{Methods}

This retrospective case series include adults 18 years and older with confirmed COVID-19 infection who were admitted between March $17^{\text {th }}$ and April $17^{\text {th }}$ and developed evidence of barotrauma (subcutaneous emphysema, pneumothorax or pneumomediastinum) on plain chest radiography or computed tomography (CT) scan. A single thoracic surgery service active in two of New York City Boroughs; Brookdale University Hospital in Brooklyn and Jamaica Hospital in Queens, was involved in the management of these cases.

Information about general population demographic and mechanical ventilation setting and outcomes were collected. In all cases diagnosis of COVID-19 infection was confirmed with reverse transcriptase polymerase chain reaction assays performed by nasopharyngeal swab. Data was extracted from electronic records.

\section{Results}

A total of 25 patients with barotrauma secondary to COVID-19 respiratory failure were managed by A single Thoracic Surgery service. The average age at the time of diagnosis 60.1 years (Table 1). 14 out of 25 patients were over the age of 60 . The majority of these patients had underlying comorbidities, with HTN and DM being the most common. 9 (36\%) suffered from Obesity and had a BMI above $30.64 \%$ of patients who developed barotrauma died and the rest still require intensive care.

All these patients were managed by lung protective strategies. $12(48.0 \%)$ of these patients had evidence of severe ARDS at the time of the barotrauma ( $\mathrm{PaO} 2 /$ FiO2<100) (Table 2), 10 with moderate ARDS (PaO2/ FiO2 of 100-200). Positive end expiratory pressure (PEEP) requirements were between $10-20 \mathrm{cmH} 20$ with a mean of $14.2 \mathrm{cmH} 20$, the median CRP 20.

The most common manifestation of barotrauma is overt pneumothorax, as 14 (56. \%) developed Pneumothorax (Table 3) and 7 (28\%) had evidence of subcutaneous emphysema on Xray and physical exam. 15 (60\%) required

Table 1: Patients Demographics

\begin{tabular}{|l|c|}
\hline \multicolumn{2}{|c|}{ Demographics } \\
\hline Age (mean \pm SD) & $60.1 \pm 9.9$ \\
\hline Ethnicity: Hispanic & $15(60.0 \%)$ \\
\hline Comorbid conditions \\
\hline Obesity & $7(36.0 \%)$ \\
\hline DM & $10(52.6 \%)$ \\
\hline HTN & $9(47.0 \%)$ \\
\hline COPD/Asthma & $4(21.0 \%)$ \\
\hline Highest BUN/Cr (median, [Range]) & $63[15-212] / 4[0.74-10.8]$ \\
\hline WBC (median [range]) & $14.1[1.9-29.4]$ \\
\hline CRP (median [range]) & $20[4.8-39.8]$ \\
\hline
\end{tabular}

Age in years, SD: standard deviation, DM: Diabetes mellitus, HTN: hypertension, COPD: chronic obstructive pulmonary disease, BUN: Blood urea nitrogen, $\mathrm{Cr}$ : creatinine, WBC: white blood cell count, CRP: c-reactive protein. 
Table 2: Severity of ARDs and outcomes.

\begin{tabular}{|c|c|}
\hline \multicolumn{2}{|c|}{ ARDS (n,\%) } \\
\hline Mild & $3(12.0 \%)$ \\
\hline Moderate & $10(40.0 \%)$ \\
\hline Severe & $12(48.0 \%)$ \\
\hline Mortality (n, \%) & $16(64.0 \%)$ \\
\hline Critically III (n,\%) & $9(36.0 \%)$ \\
\hline
\end{tabular}

ARDS: Acute respiratory distress syndrome

Table 3: Barotrauma findings and associated PEEP

\begin{tabular}{|l|c|c|}
\hline \multicolumn{3}{|c|}{ Barotrauma findings and associated PEEP } \\
\hline & PEEP 10-15 & PEEP >15 \\
\hline Subcutaneous emphysema $(\mathrm{n})$ & 3 & 4 \\
\hline Pneumothorax $(\mathrm{n})$ & 9 & 5 \\
\hline Pneumomediastinum (n) & 3 & 3 \\
\hline
\end{tabular}

PEEP: Positive end expiratory pressure

Table 4: Time between mechanical ventilation and Barotrauma/Death Days from mechanical ventilation to barotrauma ${ }^{a}$

\begin{tabular}{|l|c|}
\hline Overall (median [Range]) & $3.5[0-15]$ \\
\hline Days 0-3 (n,\%) & $11(57.8 \%)$ \\
\hline Days 4-15 $(n, \%)$ & $8(42.1 \%)$ \\
\hline
\end{tabular}

\section{Barotrauma to Death in days}

\begin{tabular}{l|l}
\hline Overall (median [range]) & 3 [1-12]
\end{tabular}

Days 0-3 (n,\%) $6(50 \%)$

Days $4-12(n, \%) \quad 6(50 \%)$

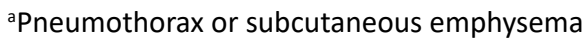

thoracostomy tube insertion, all patients who developed pneumothorax underwent bedside insertion of tube thoracostomy. Median PEEP in the patient who underwent tube thoracostomy is 14 , and 9 of these patients died. The median time for development of pneumothorax 3.5 days (table 4) and $40 \%$ of these happened within one day of mechanical ventilation.

Six patients had manifestations of barotrauma before starting mechanical ventilation (findings of pneumomediastinum, subcutaneous emphysema, or both). These patients were observed strictly with clinical exams and serial chest X-rays. Pneumomediastinum was found on Computer topography (CT) scan done to rule out Pulmonary embolism (PE) in 2 of these patients as they had an elevated D-dimer. In this group 3 patient were found to have spontaneous pneumomediastinum and 3 patients developed barotrauma after they were started on noninvasive ventilation.

\section{Discussion}

The COVID-19 pandemic began in late 2019 in China. It rapidly spread to Europe, the United States and other parts of the world. It is a highly contagious viral infection with a wide range of symptoms, from asymptomatic disease to respiratory failure, ARDS, and death with a high mortality rate $^{18-20}$. In COVID- 19, patients' respiratory failure can progress rapidly, requiring mechanical ventilation due to severe hypoxemia and ventilator challenges akin to those seen in ARDS. ARDS NET management recommendations have been the standard employed in the management of such cases.

High PEEP is often used as part of the ventilator management strategy. This is known to be associated in some cases with barotrauma requiring thoracostomy drainage. In such patients, this complication can cause profound hemodynamic changes and is associated with a higher mortality rate.

This study presents the findings of a single Thoracic Surgery service functioning at two medical centers in the New York City boroughs of Brooklyn and Queens. New York City has experienced the unfortunate distinction of becoming the COVID-19 epicenter in the United States. The two of New York's five boroughs experiencing the most significant numbers of COVID-19 cases are Queens and Brooklyn, respectively ${ }^{21}$. The large population density and particular demographic characteristics of these two boroughs have proven a fertile area for the spread of this infection. This has resulted in experiencing a large volume of the most severe cases. Subsequently, this has led to experiencing a seemingly large number of cases of barotrauma often requiring additional life-saving invasive procedures.

This series demonstrates the majority of cases were mechanically ventilated with findings of pneumothorax or extensive subcutaneous emphysema. This is associated with severe lung injury and an even higher mortality than previously described in COVID-19 patients requiring mechanical ventilation without barotrauma. The mortality rate associated with COVID-19 barotrauma is higher (64\%) compared to (25-50\%) in patient who required mechanical ventilation but didn't develop barotrauma. While this result is not likely an independent predictor of mortality it may reflect a worse disease process and prognosis.

In our patient group, 3 patients found to have spontaneous pneumomediastinum or pneumothorax. Similar case reports from China also described spontaneous pneumomediastinum in COVID19 patients ${ }^{16}$. These patients presented with spontaneous pneumomediastinum or pneumothorax seen early in the course of the disease before starting mechanical ventilation, which may reflect a severe form of lung injury associated with COVID-19 infection. This might be underestimated as evidence of barotrauma was only noted on Chest CT obtained to rule out other pathology and was not seen on CXR in 2 cases.

Anzueto et al found that barotrauma is more likely to present in patient with underlying lung disease (ARDS, Interstitial lung disease or asthma) and was associated with a higher mortality compared to mechanically ventilated patient without evidence of barotrauma (51.4\% 
Vs $39.2 \%)^{13}$. The relative risk of increased mortality attributable to barotrauma is $31.1 \%$. In a prospective observational multicenter study analyzing pneumothorax in the intensive care unit; Lassence et al reported a median of 4 days between mechanical ventilation and iatrogenic pneumothorax caused by ventilator injury ${ }^{22}$. In our case series we report a median of 3.5 days between the incidence of barotrauma and mechanical ventilation.

With COVID-19, barotrauma may develop and progresses rapidly after mechanical ventilation, which may reflect the worsening of spontaneous barotrauma from lung injury as observed in some of our patients. This may also reflect the severity of acute lung injury from the SARS-CoV-2 infection. In our cohort, $84 \%$ of the patients developed barotrauma within the first week. Only three patients developed barotrauma after 1 week.

There is also the added management problem of limiting the risk to the providers performing invasive procedures associated with barotrauma. It has been postulated that the SARs-CoV-2 virus will be aerosolized during procedures involving the aerodigestive tract ${ }^{23-25}$. In the instance that barotrauma progresses to pneumothorax, a percutaneous thoracostomy tube is the authors' preferred treatment of choice over standard open thoracostomy as it may reduce the exposure of healthcare providers and other patients to aerosolized particles. This approach utilizes Seldinger's technique of wire-guided, minimally invasive thoracostomy tube placement. This approach is validated and is increasingly performed ${ }^{26,27}$. As a result, we have hastened this technique into our service's approach to pneumothorax management. It is essential to take the precautions described for any aerosol-generating procedure, i.e., ensure adequate personal protective gear (Fitted respirator mask, gloves, gown, and eye protection), perform in negative pressure rooms ${ }^{28}$. As there is clear data that SARS-CoV-2 can remain viable in aerosols for three hours ${ }^{29}$ and even more stable in surfaces for 72 hours Limitations are the retrospective nature of this case review. Further research including a comparison group would be necessary to elucidate the basis of these findings better. In the absence of high-quality evidence Reports describing different presentation and complication of SARS-CoV-2 can serve as an essential guide to other physicians till better quality evidence emerges.

\section{Conclusion}

SARs-CoV-2 associated barotrauma is a common subtle complication of COVID-19 infection that's underreported and may reflect a worse prognosis. This paper presents a unique experience of barotrauma associated with the management of severe COVID-19.

COVID 19 pneumonia presents its unique ventilatory management challenges. Underlying altered lung compliance and ventilatory requirement providing high pressures put these patients at high risk of barotrauma. In select cases, especially in the absence of overt pneumothorax, they can be observed with close monitoring. In cases where a thoracostomy is required, a percutaneous approach seems more feasible and theoretically can limit exposure to the provider.

\section{References}

1. Johns Hopkins University. COVID-19 United States Cases 2020, May 14; Available from: https://coronavirus.jhu.edu/us-map.

2. Wu Z, McGoogan JM. Characteristics of and Important Lessons From the Coronavirus Disease 2019 (COVID-19) Outbreak in China: Summary of a Report of 72314 Cases From the Chinese Center for Disease Control and Prevention. JAMA. 2020; 323(13): 1239-1242.

3. Zhou F, Yu T, Cao B, et al. Clinical course and risk factors for mortality of adult inpatients with COVID-19 in Wuhan, China: a retrospective cohort study. Lancet. 2020; 395(10229): 1054-1062.

4. Goyal P, Choi JJ, Safford MM, et al. Clinical Characteristics of Covid-19 in New York City. N Engl J Med. 2020; 382:2 372-2374.

5. Slutsky AS, Ranieri VM. Ventilator-induced lung injury. N Engl J Med. 2013; 369(22): 2126-2136.

6. Walkey AJ, Goligher EC, Fan E, et al. Low Tidal Volume versus NonVolume-Limited Strategies for Patients with Acute Respiratory Distress Syndrome. A Systematic Review and Meta-Analysis. Ann Am Thorac Soc. 2017; 14(4): S271-S279.

7. Alhazzani W, Møller MH, Arabi YM, et al. Surviving Sepsis Campaign: guidelines on the management of critically ill adults with Coronavirus Disease 2019 (COVID-19). Intensive Care Med. 2020; 46(5): 854-887.

8. Fan E Del Sorbo L, Goligher EC, et al. An Official American Thoracic Society/European Society of Intensive Care Medicine/Society of Critical Care Medicine Clinical Practice Guideline: Mechanical Ventilation in Adult Patients with Acute Respiratory Distress Syndrome. Am J Respir Crit Care Med. 2017; 195(11): 1253-1263.

9. Rhodes A, Evans LE, Alhazzani W, et al. Surviving Sepsis Campaign: International Guidelines for Management of Sepsis and Septic Shock: 2016. Crit Care Med. 2017; 45(3): 486-552.

10. Yasuda H, Nishimura T, et al. Optimal plateau pressure for patients with acute respiratory distress syndrome: a protocol for a systematic review and meta-analysis with meta-regression. BMJ Open. 2017;7(5): e015091.

11. Briel MB, Meade M, Mercat A, et al. Higher versus lower positive end-expiratory pressure in acute lung injury and acute respiratory distress syndrome: systematic review and individual patient data meta-analysis. Critical Care. 2010; 303(9): 865-873.

12. Hyzy RC, Taha AR. Diagnosis, management, and prevention of pulmonary barotrauma during invasive mechanical ventilation in adults in UpToDate Waltham MA UpToDate 2020.

13. Anzueto A, Frutos-vivar F, Esteban A, et al. Incidence, risk factors and outcome of barotrauma in mechanically ventilated patients. Intensive Care Med. 2004; 30(4): 612-619.

14. Burns KE, Adhikari NK, Slutsky AS, et al. Pressure and volume limited ventilation for the ventilatory management of patients with acute lung injury: a systematic review and meta-analysis. PLoS One. 2011; 6(1): e14623.

15. Gattinoni L, Pelosi P, Suter PM, et al. Acute respiratory distress syndrome caused by pulmonary and extrapulmonary disease. Different syndromes? Am J Respir Crit Care Med. 1998; 158(1): 3-11.

16. Eisner MD, Thompson BT, Schoenfeld D, et al. Airway pressures 
and early barotrauma in patients with acute lung injury and acute respiratory distress syndrome. Am J Respir Crit Care Med. 2002; 165(7): 978-982.

17. Zhou C, Gao C, Xu M, et al. COVID-19 with spontaneous pneumomediastinum. The Lancet Infect Dis. 2020; 20(4): 510.

18. Rodriguez-Morales AJ, Cardona-Ospina JA, Gutierrez-Ocampo E, et al. Clinical, laboratory and imaging features of COVID-19: A systematic review and meta-analysis. Travel Med Infect Dis. 2020; 34: 101623.

19. Wu C, Chen X, Cai Y, et al. Risk Factors Associated With Acute Respiratory Distress Syndrome and Death in Patients With Coronavirus Disease 2019 Pneumonia in Wuhan China. JAMA Intern Med. 2020; 180(7): 934-943.

20. Weiss P, Murdoch DR. Clinical course and mortality risk of severe COVID-19. Lancet. 2020; 395(10229): 1014-1015.

21. New York City Health. COVID-19: Data. 2020 May 15; Available from: https://www1.nyc.gov/site/doh/covid/covid-19-data.page.

22. de Lassence A, Timsit JF, Tafflet M, et al. Pneumothorax in the intensive care unit: incidence, risk factors, and outcome. Anesthesiology. 2006; 104(1): 5-13.

23. Tay JK, Khoo ML, Loh WS. Surgical Considerations for Tracheostomy During the COVID-19 Pandemic: Lessons Learned From the Severe Acute Respiratory Syndrome Outbreak. JAMA Otolaryngol Head Neck Surg. 2020; 146(6): 517-518.
24. American Society for Gastrointestinal Endoscopy. JOINT GI SOCIETY MESSAGE: COVID-19 Clinical Insights for Our Community of Gastroenterologists and Gastroenterology Care Providers. 2020; Available from: https://www.asge.org/home/joint-gi-societymessage-covid-19.

25. Society of Gastrointestinal and Endoscopic Surgeons. SAGES and EAES recommendations regarding surgical response to COVID-19 crisis. 2020, March 30; Available from: https://www.sages.org/ recommendations-surgical-response-covid-19/.

26. Aziz, F, Penupolu S, Flores D. Efficacy of percutaneous pigtail catheters for thoracostomy at bedside. J Thorac Dis. 2012; 4(3): 292-5.

27. McKay T, Franklin P, Steliga M, et al. Percutaneous Catheter for Spontaneous Pneumothorax. The Cardiothoracic Surgery Network. 2014.

28. Center for Disease Control. Interim infection prevention and control recommendations for patients with suspected or confirmed coronavirus disease 2019 (COVID-19) in healthcare settings. 2020, April 13; Available from: https://www.cdc.gov/coronavirus/2019ncov/hcp/infection-control-recommendations.html\#take_ precautions.

29. van Doremalen N. Aerosol and Surface Stability of SARS-CoV-2 as Compared with SARS-CoV-1. N Engl J Med. 2020; 382(16): 15641567. 